COMUNICAÇÃO CIENTÍFICA

\title{
INÍCIO DA PRODUÇÃO DE FRUTOS DE CAGAITEIRA (Eugenia dysenterica DC) IMPLANTADA EM GOIÂNIA, GOIÁS ${ }^{1}$
}

\author{
ELI REGINA BARBOZA DE SOUZA², RONALDO VELOSO NAVES², \\ MARCOS FERNANDES OLIVEIRA ${ }^{3}$
}

RESUMO - A cagaiteira é uma planta nativa do Cerrado, adaptada às condições impostas por este bioma, principalmente de sobreviver e produzir em solos muito pobres em nutrientes e em um regime de chuvas com um período acentuado de baixa precipitação. A planta é rústica, ornamental e com alta tolerância ao fogo. Seus frutos são apreciados ao natural e utilizados nos mais diversos tipos de alimentos processados. No entanto, pouco se conhece sobre quanto tempo esta espécie demora a entrar na fase reprodutiva. Este trabalho propõe-se a avaliar o início da produção de frutos de cagaiteiras implantadas na área experimental da Escola de Agronomia, Universidade Federal de Goiás-Goiânia, Goiás, Brasil, entre 2003 e 2008, quando as plantas apresentavam de cinco a dez anos de idade. Para a implantação do experimento, foram coletados frutos em dez áreas da região sudeste do Estado de Goiás e plantadas em um desenho de blocos casualizados, com uma planta por parcela, em quatro blocos, em espaçamento de $6,0 \mathrm{~m}$ x 6,0 m. As cagaiteiras apresentaram alta desuniformidade para iniciar sua produção. No quinto ano após o plantio, somente 5,2\% das plantas entraram em produção e, após dez anos, $55,7 \%$ das plantas. Destas plantas, somente $6,8 \%$ conseguiram produzir em, pelo menos, quatro anos de observação. Apenas quatro plantas entraram em produção e mantiveram esta nos seis anos de observação. O número de frutos por planta é muito baixo; somente $3,4 \%$ das plantas produzem mais de 200 frutos no décimo ano. Existe uma tendência de aumento de número de frutos com a idade da planta.

Termos para indexação: frutíferas do Cerrado, mirtáceas, produtividade.

\section{INITIAL FRUITING OF THE CAGAITA TREE (Eugenia dysenterica DC) CULTIVATED IN GOIÂNIA, GOIÁS, BRAZIL}

\begin{abstract}
The cagaita tree is a native plant of the Cerrado and adapted to the conditions of this biome, having as main characteristics the ability of growing and surviving in nutrient-poor soils and under a rainfall pattern with a pronounced low precipitation period. This is a rustic, ornamental, and highly fire-resistant plant. Its fruits are edible raw and used in many different types of processed foods. However, little is known about how long this species takes to enter the reproductive phase. This study is intended to assess the initial fruiting of cagaita plants cultivated in the experimental area of the School of Agronomy of the Federal University of Goiás, Goiânia, Brazil, between 2003 and 2008, when the cagaita plants were five to ten years old. For the experiment, fruits were collected from ten areas of the Southeast region of the State of Goiás and planted in a randomized block design with one plant per plot in four blocks, at $6 \mathrm{~m} \mathrm{x} 6 \mathrm{~m}$ spacing. The cagaita plants showed a great lack of uniformity to begin to fruit. In the fifth year after planted out, only $5.2 \%$ of the plants began to fruit and after ten years, this number increased to $55.7 \%$. Among these plants, only $6.8 \%$ fruited during at least four years of observation. Only four plants entered the fruiting phase and remained so during the six years of observation. The number of fruits per plant is very low; only $3.4 \%$ of the plants produced more than 200 fruits in the tenth year. The study showed that the number of fruits tends to increase as the plant gets older.
\end{abstract}

Index terms: Cerrado fruit, Myrtaceae, productivity.

${ }^{1}$ (Trabalho 154-12). Recebido em: 16-05-2013. Aceito para publicação em: 09-09-2013.

${ }^{2}$ Eng $^{\circ}$. Agr ${ }^{\circ}$., Doutor em Agronomia. Professor Adjunto, Escola de Agronomia/UFG - Rodovia Goiânia/Nova Veneza km 0, CEP 74001-970, Campus Samambaia-E-mail: eliregina1@gmail.com; ronaldo@agro.ufg.br

${ }^{3} \mathrm{Eng}^{\mathrm{o}}$. Agr ${ }^{\mathrm{o}}$., Doutor em Agronomia. E-mail: marcoseherika@yahoo.com.br 
A cagaiteira (Eugenia dysenterica) é uma espécie frutífera nativa do Cerrado brasileiro pertencente à família Myrtaceae. Ainda é pouco utilizada, mesmo nesta região, apesar de seu grande potencial. É encontrada em quase toda a extensão do Cerrado, principalmente em sua área nuclear. Apresenta comportamento bem peculiar quanto à ocorrência, pois aparece com alta frequência em algumas regiões, formando aglomerações. As cagaiteiras podem atingir mais de $10 \mathrm{~m}$ de altura, embora a grande maioria apresente porte entre $4 \mathrm{~m}$ e $8 \mathrm{~m}$; as folhas são caducas e seu tronco é muito sulcado, com forte presença de cortiça. Apresenta grande adaptação em ambientes de solos pobres em nutrientes, com elevada acidez, presença de alumínio tóxico e com grande déficit hídrico em alguns meses do ano (NAVES et al., 2002). A cagaiteira apresenta tanto autofecundação quanto fecundação cruzada, sendo a polinização realizada principalmente pelas mamangavas das espécies Bombus atratus e B. morio, no período da manhã (PROENÇA; GIBBS, 1994). A duração média do início da formação dos frutos até a maturação é de aproximadamente, quatro semanas, sendo a maturação dos frutos relativamente rápida e coincidente com o início do período chuvoso no Cerrado. A dispersão da cagaiteira logo no início do período chuvoso parece ser imprescindível para que, após sua germinação, exista um período favorável de estabelecimento e crescimento, permitindo a sobrevivência da plântula no período seco subsequente (SANO et al., 1995).

A cagaiteira é considerada grande produtora de frutos; além disso, tem potencial para utilização como planta ornamental, como fornecedora de cortiça, e sua madeira pode ser empregada para obras da construção civil, lenha e carvão. Alguns trabalhos relacionados aos óleos essenciais (DUARTE et al., 2009; 2010) e genética e melhoramento (AGUIAR et al., 2009; 2011; CHAVES et al., 2011) têm sido abordados recentemente. No entanto, não se tem conhecimento, em condição de cultivo, sobre a duração da fase imatura até a cagaiteira de pé-franco entrar na fase reprodutiva. O presente trabalho teve como objetivo avaliar o início da produção de frutos em cagaiteiras implantadas a partir de sementes, em Goiânia-GO.

O experimento foi conduzido na área experimental da Escola de Agronomia, da Universidade Federal de Goiás (UFG) (latitude 16 $35^{\prime} 38^{\prime \prime} \mathrm{S}$, longitude $49^{\circ} 17^{\prime} 24^{\prime}$ ' W Gr, a $730 \mathrm{~m}$ de altitude), Goiânia, Goiás, no período de outubro de 2003 a outubro de 2008, quando as plantas apresentavam de cinco a dez anos de idade, respectivamente. Para a implantação do experimento, foram coletados frutos em dez áreas da região sudeste do Estado de Goiás e plantadas em um desenho de blocos casualizados, com uma planta por parcela, em quatro blocos, em espaçamento de $6,0 \mathrm{~m}$ x 6,0 m. O clima do local é classificado como Tropical Chuvoso $\left(\mathrm{A}_{\mathrm{w}}\right)$ no Sistema Internacional de Köppen. O solo da área experimental da EA/UFG foi classificado como Latossolo Vermelho.

A altura média das plantas, aos cinco anos, foi de $1,98 \mathrm{~m}$, com diâmetro médio, a dez $\mathrm{cm}$ do solo, de $0,042 \mathrm{~m}$, e aos dez anos a altura média foi de $4,19 \mathrm{~m}$, com diâmetro médio de $0,102 \mathrm{~m}$. No quinto ano após o plantio no campo, 23 plantas iniciaram a produção e, assim, sucessivamente, com 13; 59; 56; 17 e 77 plantas até o décimo ano após o plantio. Dez anos após o plantio, 245 plantas de um total de $440(55,7 \%)$ haviam passado para a fase reprodutiva, produzindo frutos em, pelo menos, um ano. Portanto, verifica-se que a mudança da fase vegetativa para a fase reprodutiva na cagaiteira é demorada e desuniforme. Este fato merece atenção, no sentido de se criar condição para aumentar a uniformidade e favorecer seu desenvolvimento a campo. $\mathrm{O}$ uso da irrigação, aliada à cobertura morta no período da estiagem, que se estende normalmente dos meses de abril a setembro no Cerrado, pode ser uma boa ferramenta para minimizar a desuniformidade inicial das plantas.

Observa-se que o número de plantas em produção é muito variável nos seis anos de estudo (Figura 1), e bastante baixo em relação ao total de plantas. Este comportamento de grande variação do número de plantas em produção e o período que levou para estas entrarem na fase reprodutiva, pode concorrer para dificultar iniciativas de cultivo desta espécie. Especula-se que, nos anos iniciais do plantio a campo, principalmente no primeiro e segundo anos, seria necessário um aporte maior de tecnologia visando à maior uniformidade e desenvolvimento dessas plantas.

Poucas plantas entraram em produção e mantiveram esta por mais de um ano (Figura 2). Somente quatro plantas conseguiram entrar em produção no quinto ano após o plantio a campo e manter esta produção nos seis anos de observação. Observa-se, que além de poucas plantas entrarem em produção após o quinto ano de seu plantio no campo, o número de frutos é muito baixo, indicando uma entrada em produção lenta, tanto em número de plantas quanto em número de frutos (Tabela 1). Cagaiteiras com maior diâmetro de caule tendem a produzir um número expressivo de frutos (SOUZA et al., 2008), porém quando se trabalha com plantas em seu ambiente natural, não se conhece sua idade. No entanto, pode-se estimá-la considerando que as plantas de maior porte sejam mais antigas.

Conclui-se que as cagaiteiras em estudo apresentam alta desuniformidade para iniciar sua produção. Existe uma tendência de aumento de número de frutos com a idade da planta. 


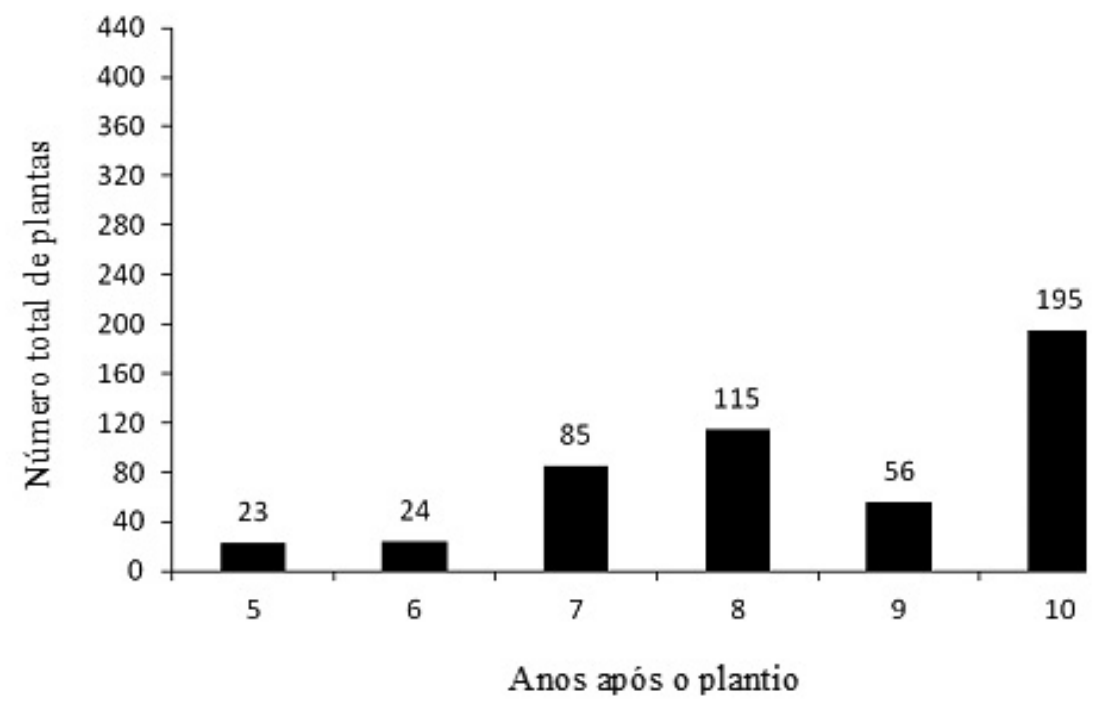

FIGURA 1-Número de cagaiteiras em produção, de um total de 440 plantas, em cada ano do estudo. Goiânia-GO. 2008.

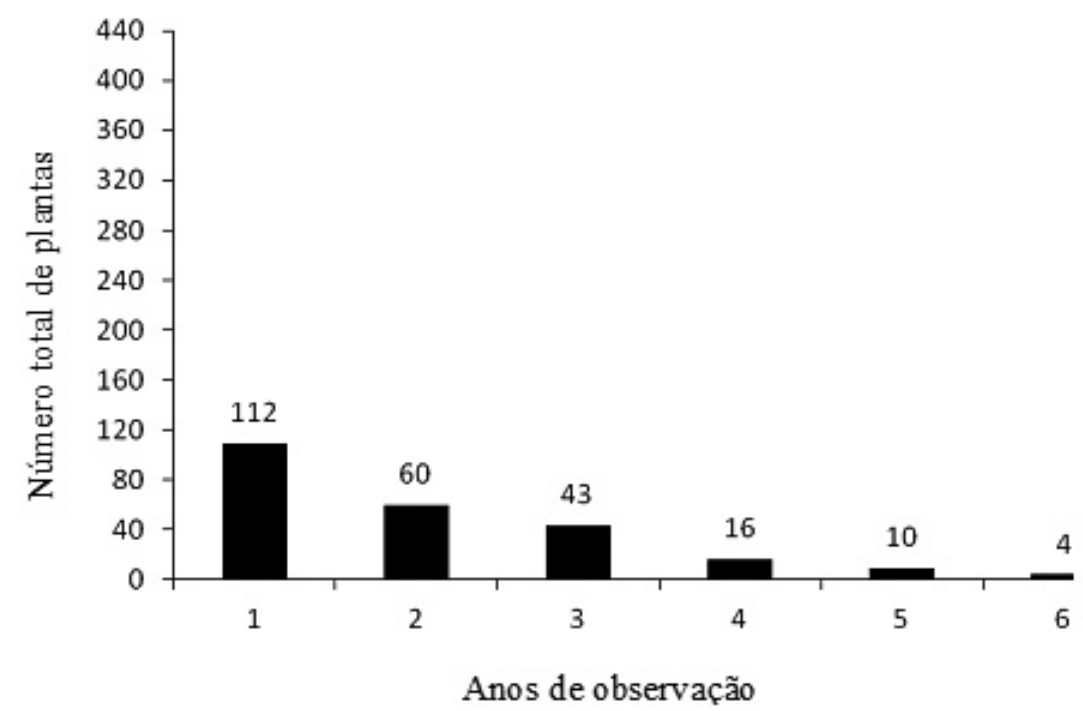

FIGURA 2- Plantas de cagaita, de um total de 440 plantas, que produziram frutos em um, dois, três até seis anos consecutivos. Goiânia-GO. 2008 


\section{REFERÊNCIAS}

AGUIAR, A. V.; VENCOVSKY, R.; CHAVES, L. J.; MOURA, M. F.; MORAIS, L. K. Genetics and expected selection gain for growth traits in Eugenia dysenterica DC. populations. Bragantia, Campinas, v. 68, n. 3, p. 629-637, 2009.

AGUIAR, A. V.; MOURA, N. F.; MOURA, M. F.; ZUCCHI, M. I.; VENCOVSKY, R.; CHAVES, L. J. Relação entre variação genética de caracteres quantitativos e marcadores moleculares em subpopulações de cagaiteiras (Eugenia dysenterica DC.). Revista Brasileira de Fruticultura, Jaboticabal, v. 33, n. 1, p. 157-169, 2011.

CHAVES, L. J.; VENCOVSKY, R.; SILVA, R. S. M.; TELLES, M. P. C.; ZUCCHI, M. I.; COELHO, A. S. G. Estimating inbreeding depression in natural plant populations using quantitative and molecular data. Conservation Genetics, Dordrecht, v. 12, n. 2, p.569-576, 2011.

DUARTE, A. R.; NAVES, R. V.; SANTOS, S. C.; SERAPHIN, J. C.; FERRI, P. H. Seasonal influence on the essential oil variability of Eugenia dysenterica. Journal of the Brazilian Chemical Society, São Paulo, v. 20, n. 5, p.967-974, 2009.

DUARTE, A. R.; NAVES, R. V.; SANTOS, S. C.; SERAPHIN, J. C.; FERRI, P. H. Seasonal influence on the essential oil variability of Eugenia dysenterica. Journal of the Brazilian Chemical Society, São Paulo, v. 21, n. 8, p.1.459-1.467, 2010.

NAVES, R. V.; BORGES, J. D.; CHAVES, L. J. A cagaiteira. Revista Brasileira de Fruticultura, Jaboticabal, v. 24, n. 2, 2002. Capa.

PROENÇA, C. E. B.; GIBBS, P. E. Reproductive biology of eight sumpatric Myrtaceae from Central Brazil. New Phytologist, Oxford, v. 126, p. 343354, 1994.

SANO, S. M.; FONSECA, C. E. L. da; RIBEIRO, J. F.; OGA, F. M.; LUIZ, A. J. B. Folhação, floração, frutificação e crescimento inicial da cagaiteira. Pesquisa Agropecuária Brasileira, Brasília, v. 30, n. 1, p. 5-14, 1995.

SOUZA, E. R. B. de; NAVES, R. V.; BORGES, J. D.; VERA, R.; FERNANDES, E. P.; SILVA, L. B.; TRINDADE, M. G. Fenologia de cagaiteira (Eugenia dysenterica DC.) no Estado de Goiás. Revista Brasileira de Fruticultura, Jaboticabal, v. 30, n. 4, p. 1009-1014, 2008. 